\title{
H2BC4 Gene
}

National Cancer Institute

\section{Source}

National Cancer Institute. H2BC4 Gene. NCI Thesaurus. Code C162924.

This gene is involved in histone complex formation. 